\title{
939 AUTOCRINE/PARACRINE FACTORS IN CANCER ASSOCIATED FIBROBLASTS DRIVE IMMUNOSUPPRESSION THROUGH IMPAIRED MYELOID CELL FUNCTIONS
}

Nikita Sharma*, Priya Govindaraju, Shermineh Bradford, Yarong Wang, Brianna Flynn, Candace Wai Sze Lei, Varenka Rodriguez Di Blasi, Jeanine Pignatelli, Kang Liu, Emily Corse, Abhishek Kashyap. Boehringer Ingelheim Pharmaceuticals, Ridgefield, CT, USA

Background Cancer associated fibroblasts (CAFs) promote tumorigenesis by secreting immunosuppressive cytokines, stimulating angiogenesis, and supporting the growth of tumor cells. Through their interactions with immune cells, CAFs are known to directly impact the functionality of $\mathrm{T}$ cells and macrophages. However, CAF interaction with dendritic cells (DCs) and DC progenitor cells and its impact on DC function is relatively understudied and was the main focus of this study.

Methods Two types of coculture systems were used in this study. For the human system, fibroblasts from lung squamous cell carcinoma (LUSC) were cocultured with MUTZ3 cells (hematopoietic progenitor cells) in the presence of DC differentiation stimuli, sometimes followed by DC maturation stimuli. For the mouse coculture system, activated (YPSC-c) and inactivated (PSC-b) pancreatic stellate cells (PSCs) were isolated from the pancreas of $\mathrm{C} 57 \mathrm{BL} / 6$ mice by the density gradient method and co-cultured in the presence of bone marrow cells in the presence of DC differentiation and maturation stimuli. For human tumor antigen processing and cross presentation assay MART1 peptide (10mer and 20mer) was used.

Results Co-culture of human and murine hematopoietic progenitor cells with fibroblasts (human LUSC CAFs and murine PSC results in decrease in differentiation and maturation of DCs. DCs differentiated and matured in the presence of fibroblasts have impaired ability to process and present tumor antigen to $\mathrm{T}$ cells. In the presence of PSC fibroblasts DC differentiation from murine bone marrow cells is skewed more towards MDSC and macrophages. In contrast to inactivated PSC-b, activated PSC-c influence DC differentiation in a contact dependent manner. Furthermore, PSC-b and PSC-c show transcriptionally distinct signatures which translate to unique secretory profiles as measured by Luminex. Analysis of the conditioned media from the coculture demonstrated that PSC-c secrete (among others) CXCL1, IL6, and CCL5 chemo/ cytokines. These and other factors may play an important role in mediating fibroblast induced suppression of DC differentiation from monocytes.

Conclusions Our study demonstrates that cancer associated fibroblasts, or their precursors directly impact DC differentiation and antigen presentation via cytokines that could be targeted therapeutically to improve DC expansion and activity in the tumor microenvironment.

http://dx.doi.org/10.1136/jitc-2021-SITC2021.939 